\title{
Rat bite fever: Complex triple valve surgery for endocarditis and sinus of Valsalva fistula
} \author{
Wis \\ From the ${ }^{\mathrm{a}}$ Department of Surgery and ${ }^{\mathrm{b}}$ Division of Pediatric Cardiothoracic Surgery, Medical College of \\ Wisconsin and Herma Heart Center and Children's Hospital of Wisconsin, Milwaukee, Wis. \\ Disclosures: Authors have nothing to disclose with regard to commercial support. \\ Received for publication Aug 3, 2016; revisions received Oct 6, 2016; accepted for publication Oct 8, 2016; \\ available ahead of print Dec 2, 2016. \\ Address for reprints: Ronald K. Woods, MD, PhD, Herma Heart Center and Children's Hospital of Wisconsin, \\ 9000 W Wisconsin Ave, Milwaukee, WI 53226 (E-mail: RWoods@chw.org). \\ J Thorac Cardiovasc Surg 2017;153:e45-6 \\ $0022-5223 / \$ 36.00$ \\ Copyright (c) 2016 by The American Association for Thoracic Surgery \\ http://dx.doi.org/10.1016/j.jtcvs.2016.10.037
}

Maher N. Abadeer, MD, ${ }^{\mathrm{a}}$ Peter C. Kouretas, MD, PhD, ${ }^{\mathrm{a}, \mathrm{b}}$ and Ronald K. Woods, MD, PhD, ${ }^{\mathrm{a}, \mathrm{b}}$ Milwaukee,
We present a rare case of rat bite fever and infective endocarditis (IE) involving a sinus of Valsalva fistula and ventricular septal defect (VSD) patch.

\section{CLINICAL SUMMARY}

A 19-year-old man with previous (infant) transannular patch repair of tetralogy of Fallot was transferred to our facility with fever, chest pain, myalgia, and weight loss 1 month after a rat bite. Transthoracic echocardiography demonstrated a large fistula between the right sinus of Valsalva and the right ventricle (RV) with significant diastolic flow reversal in the thoracic aorta, vegetations on the VSD patch and tricuspid valve; severe pulmonary valve insufficiency and RV dilation, moderate tricuspid insufficiency, diminished RV function, and normal left ventricular function. Blood cultures remained negative, so he was taken for surgical repair.

Operative findings included a large fistula $(1 \mathrm{~cm}$ in diameter) below the right coronary ostium communicating with the RV with a second smaller fistula (3 $\mathrm{mm}$ in diameter) below the annulus of the right coronary leaflet and vegetation and abscess on and around the VSD patch and septum extending to the middle portion of the right coronary leaflet and the septal leaflet of the tricuspid valve. Removal of all diseased tissue resulted in (1) a large left ventricular outflow tract (LVOT) defect extending from below the resected VSD patch across the aortic annulus to just under the right coronary os, (2) a small remnant of free margin right aortic leaflet; and (3) approximately $60 \%$ of residual tricuspid septal leaflet (Figure 1). The LVOT was reconstructed with a single bovine pericardial patch imbricated at the level of the aortic annulus to create a neoannulus and extended to close the sinus defect. The aortic valve was replaced with a 23-mm mechanical prosthesis. The septal leaflet of the tricuspid valve was repaired with a bovine pericardial patch and supported by annuloplasty. Although it was not involved in the endocarditic process, the pulmonary with rat bite fever. for 2 weeks.

\section{DISCUSSION} is present. ${ }^{1}$

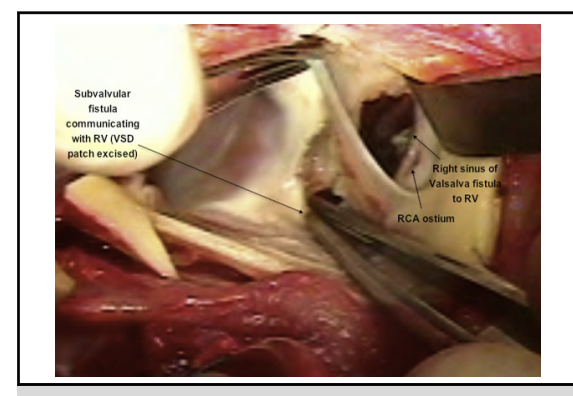

Surgeon's view demonstrating sinus of Valsalva fistulae.

\section{Central Message}

Endocarditis associated with a ventricular septal defect patch and sinus of Valsalva fistula is a rare entity. In our case, it required extensive reconstructive surgery.

See Editorial Commentary page e47.

valve was replaced with a $27-\mathrm{mm}$ bioprosthetic valve. Completion transesophageal echocardiogram demonstrated good tricuspid, aortic, and pulmonary valve functions, with no residual VSD and a widely patent LVOT. Operative cultures revealed Streptobacillus moniliformis, consistent

The postoperative course was notable for transient high-output renal failure not requiring dialysis and the need for mechanical ventilation until postoperative day 5 . He was bridged to a regimen of warfarin with aspirin and discharged on postoperative day 11 with intravenous penicillin for 6 weeks and gentamicin synergy

Rat bite fever is a rare entity, with only 17 cases of IE reported in the literature. ${ }^{1}$ The culprit is typically $S$ moniliformis, a fastidious gram-negative bacillus identifiable on culture with blood agar or by polymerase chain reaction. ${ }^{1}$ It is caused by the bite of an infected rodent or ingestion of contaminated food and characterized by fever, arthralgia, and rash. ${ }^{2}$ Progression to sepsis and end-organ dysfunction has been described, with IE representing the most lethal form of disease, especially if underlying vascular disease 


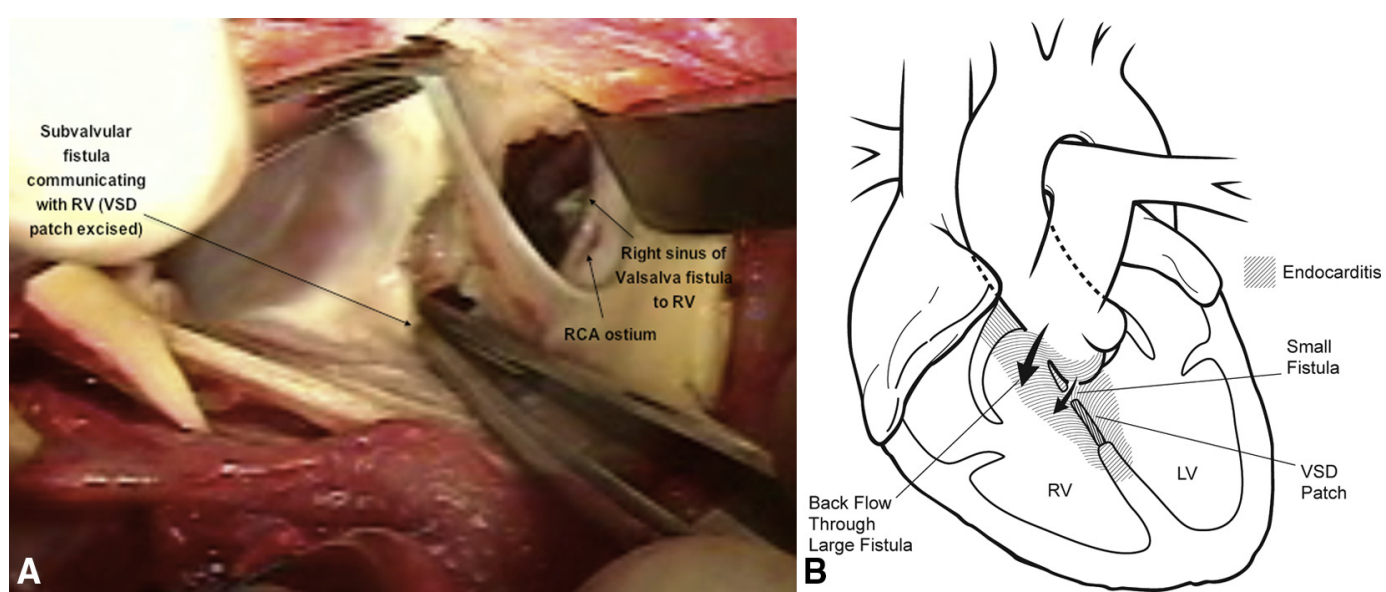

FIGURE 1. A, Intraoperative transaortic view demonstrating 2 sinus of Valsalva fistulae and destruction of the aortic annulus. B, Medical illustration of the fistulae and extent of endocarditis, as indicated by the shaded area. $R V$, Right ventricle; $V S D$, ventricular septal defect; $R C A$, right coronary artery; $L V$, left ventricle.

Surgical treatment of valvular IE requires resection and débridement of all involved tissue. If this is done correctly, either a mechanical prosthesis or a homograft can be expected to provide similar freedom from recurrent IE. ${ }^{3}$ In younger children, the Ross procedure is an attractive option, and it is recommended for children and adolescents with extensive aortic IE in the 2015 European Society of Cardiology guidelines. ${ }^{3}$ The American College of Cardiology and American Heart Association guidelines state that the Ross procedure may be considered, as long as it is performed by an experienced surgeon, in young patients for whom anticoagulation is undesirable. ${ }^{4}$ The Ross procedure was clearly not an option in our case because of the lack of a suitable pulmonary valve.

When feasible, the first line approach for atrioventricular valves is repair. For mitral valves, there is class 1, level B evidence in favor of attempting repair, keeping unaffected chordae and papillary muscles to preserve left ventricular function. ${ }^{3}$ Tricuspid valves should similarly undergo attempted repair before replacement. ${ }^{3,5}$ For patients who are unlikely to comply with medical therapy and have a valve not suitable for repair, bioprosthetic replacement is an appropriate option.

For extremely unusual cases such as ours, it is important to adhere to basic principles: completely eradicate the disease, simplify to the extent possible any prolonged extensive surgery that is anticipated; and ensure that no residual hemodynamic lesions remain. Choice of specific materials for valve repair or LVOT reconstruction is not a critical factor. Repair of IE associated with a sinus of Valsalva fistula or repair of VSD patch IE has been described in case reports or small series; however, repair involving both a sinus of Valsalva fistula and a VSD patch is exceedingly rare.

\section{References}

1. Chen PL, Lee NY, Yan JJ, Yang YJ, Chen HM, Chang CM, et al. Prosthetic valve endocarditis caused by Streptobacillus moniliformis: a case of rat bite fever. J Clin Microbiol. 2007;45:3125-6.

2. Elliott SP. Rat bite fever and Streptobacillus moniliformis. Clin Microbiol Rev. 2007:20:13-22.

3. Habib G, Lancellotti P, Antunes MJ, Bongiorni MG, Casalta JP, Del Zotti F, et al. 2015 ESC Guidelines for the management of infective endocarditis: The Task Force for the Management of Infective Endocarditis of the European Society of Cardiology (ESC). Endorsed by: European Association for Cardio-Thoracic Surgery (EACTS), the European Association of Nuclear Medicine (EANM). Eur Heart J. 2015;36:3075-128.

4. Nishimura RA, Otto CM, Bonow RO, Erwin JP III, Guyton RA, O'Gara PT, et al; ACC/AHA Task Force Members. 2014 AHA/ACC Guideline for the Management of Patients With Valvular Heart Disease: executive summary: a report of the American College of Cardiology/American Heart Association Task Force on Practice Guidelines. Circulation. 2014;129:2440-92.

5. Neely RC, Leacche M, Shah J, Byrne JG. Current readings: status of surgical treatment for endocarditis. Semin Thorac Cardiovasc Surg. 2014;26:53-66. 